\title{
Análisis de la información internacional en la prensa digital española
}

\author{
Juan Antonio García Galindo \\ Universidad de Málaga \\ jagarciag@uma.es \\ Aida María de Vicente DomíngueZ \\ Universidad de Málaga \\ amdevicente@gmail.com
}

Recibido: 15 de noviembre de 2013

Aceptado: 12 de febrero de 2014

\begin{abstract}
Resumen
La presente investigación analiza las noticias publicadas en la sección internacional de la prensa digital española con el fin de conocer cuales son los acontecimientos que se convierten en información de actualidad, las zonas geográficas que tienen presencia mediática, y el tipo de fuentes que se usan para elaborar las piezas informativas. La metodología ha consistido en analizar el corpus constituído por todas las noticias publicadas en la sección internacional de elpaís.es $(\mathrm{n}=1.235)$ durante el primer trimestre de 2012. Este estudio intenta mostrar cuál es la información internacional que publica la prensa española, y el modo como los medios tratan informativamente esa realidad.
\end{abstract}

Palabras clave: periodismo, información, internacional, acontecimiento, prensa digital.

\section{Analysis of international information in Spanish digital newspaper}

\begin{abstract}
The following research analyzes the news that were published on the international section of digital Spanish newspapers in order to know which events can be converted into present time information, the areas that have media presence, and the kind of sources that are used to elaborate informative pieces. The methodology consisted on a corpus formed by news that were published on elpais.es international section $(n=1.235)$ during the first semester of 2012. This study tries to show which international information is published by Spanish newspapers, and the way those media treat that reality informatively.
\end{abstract}

Keywords: journalism, information, international, event, digital newspaper

\section{Referencia normalizada}

GARCÍA GALINDO, Juan Antonio y VICENTE DOMÍNGUEZ, Aida María de (2014): “Análisis de la información internacional en la prensa digital española". Estudios sobre el Mensaje Periodístico. Vol. 20, Núm. 1 (enero-junio), págs.: 85-94. Madrid, Servicio de Publicaciones de la Universidad Complutense.

Sumario: 1. Introducción. 2. Metodología. 3. Resultados; 3.1. Localización de la información internacional; 3.2. Temáticas de las noticias internacionales; 3.3. Cobertura de los acontecimientos; 3.4. Las fuentes. 4. Conclusiones. 5. Referencias bibliográficas.

\section{Introducción}

El estudio de los acontecimientos que jalonan la actualidad internacional reviste un especial interés porque a través de ellos se va configurando el presente y, a su vez, la historia. Como escribiera Mead (1932), el mundo es un mundo de acontecimientos. Éstos están en el origen de las transformaciones que se van produciendo en el mundo en los ámbitos económico, político, o sociológico. Del mismo modo que la información internacional ofrecida por los medios de comunicación es el escenario al que miramos los ciudadanos para saber qué ocurre en el mundo. Aunque, como explica McCombs 
(2006), no hay suficientes periodistas para cubrir todos los hechos que se producen cada día.

En este sentido, el objeto de la presente investigación es averiguar qué registros temáticos internacionales interesan a la prensa española, con el fin de conocer cuales son los acontecimientos que se convierten en noticia y que adquieren la categoría de actualidad. Partimos del concepto de acontecimiento entendido como una circunstancia adscrita a un momento dado, que produce una irrupción en el presente provocando una ruptura definitiva en él o una alteración del mismo ${ }^{1}$.

Hemos considerado significativo analizar las coordenadas espacio temporales de las noticias (localización del acontecimiento) y la procedencia de las informaciones (fuentes). Como expone De la Calle, "multitud de estudios ya hacen referencia a la manera en la que los medios de comunicación construyen acontecimientos" (2010: 73), por lo que este mismo autor plantea la necesidad de avanzar en el análisis del acontecimiento y de examinar al mismo tiempo los medios.

Así, junto al análisis de esos registros temáticos, la localización geográfica y la procedencia de las informaciones, trataremos también de dar respuesta a la diferente cobertura que reciben los hechos informativos. De manera que del análisis de la agenda mediática internacional podamos conocer, de un lado, qué es lo que se considera más relevante, y de otro, la forma cómo los medios tratan informativamente esa realidad, es decir, cómo la construyen. Una representación de la realidad que responde a intereses diversos, pero que nos permite reconocer el estado del mundo a través del prisma de los medios de comunicación y, lo que es igualmente importante, reconstruirlo científicamente. Con similar propósito se refería el historiador Josep Fontana cuando hacía referencia a la importancia de las fuentes históricas: "Todo intento de avanzar respecto del estado actual de nuestros conocimientos -escribe este historiador-, ha de basarse en la confrontación con el material primario que nos proporcionan las fuentes" (Fontana, 1992: 115).

En concreto, la investigación analiza la sección internacional de la prensa digital española durante el primer trimestre del año 2012. Estudios precedentes han abordado de forma colateral o directa algunas de estas cuestiones en medios de comunicación impresos (prensa) o audiovisuales (televisión) españoles. Tómense como referencia las aportaciones ofrecidas por Moreno (2000), Campos y Martínez (2012), o Díaz (2008). Razón por la cual hemos considerado relevante abordar en esta ocasión el estudio de las ediciones digitales.

1 Nos referimos a aquellos hechos que de alguna forma provocan una ruptura con el estado que se vive en un momento en concreto. A este respecto podemos destacar que existen dos tipos: aquellos que una vez que suceden producen una ruptura definitiva con el presente; y aquellos que no lo cambian, sólo provocan una alteración de éste en un determinado espacio temporal. Por otro lado, también podemos distinguir dos tipos tomando como referencia el origen del acontecimiento: los que se producen de forma inesperada, imposibles de deducir a través de una lógica lineal, y los que tienen una explicación causal. En estos casos, el pasado suele convertirse en referente del acontecimiento. Sobre el acontecimiento pueden consultarse, entre otras, las obras de los siguientes autores: Badiou (1988); Moles (1972), Morín (1972) o Tudesq (1973). 
Navarro (2002) analizó, en diversos diarios digitales (washingtonpost.com, elpais.es, clarin.com; El universal on Lide y The Times), la geolocalización y las temáticas más representativas de los contenidos internacionales ofrecidos en sus portadas del 15 de febrero al 12 de marzo de 1999, del 7 al 18 de junio de 1999, y del 3 al 15 de enero de 2000. Ahora, más de una década después pretendemos analizar nuevamente la localización geográfica y las temáticas más recurrentes en la prensa digital española, cuyos resultados más relevantes nos pueden permitir establecer parámetros comparativos, junto a nuevas variables: fuentes y cobertura ofrecida de cada acontecimiento.

Concretado el propósito de nuestra investigación, y su vigencia y relevancia, los objetivos que se pretenden abordar con ella son los siguientes:

- Conocer las áreas geográficas con cobertura informativa, y los países de cada una de ellas con presencia mediática en la prensa española.

- Identificar la naturaleza de los acontecimientos de la actualidad internacional.

- Distinguir cuáles son los hechos que tienen mayor cobertura informativa.

- Averiguar cómo informan los medios, y si las publicaciones proceden de fuentes directas (corresponsales o enviados especiales) o de fuentes indirectas (agencias u otros medios).

Todo ello permitirá conocer la agenda y la geografía de la información internacional en los nuevos medios, así como el modo cómo tratan informativamente esta realidad. Hace ahora casi treinta años, el profesor Eliseo Verón (1979) mostraba cómo los medios de comunicación franceses convertían en acontecimiento el accidente producido en 1979 en la central nuclear estadounidense de Three Mile Island. Mediante una radiografía de los hechos y el análisis del discurso de la información de los medios consultados, Verón recompone el acontecimiento y explica la verdadera importancia y gravedad de los hechos, por encima de la sobreinformación, de la manipulación, o de la torpeza informativa.

\section{Metodología}

La metodología empleada para realizar la investigación se ha estructurado en cinco fases:

- Revisión bibliográfica: se han consultado diversas bases de datos (Dialnet, Rebiun y Teseo) para acceder a las investigaciones precedentes realizadas sobre este campo de estudio.

- Selección del medio: se ha escogido el elpais.es por ser el diario más leído en su versión impresa (según los datos aportados por la Oficina de la Justificación de la Difusión en la última oleada de 2011) y digital (tal y como señalan los resultados del informe El uso de Internet en Europa, realizado por comScore en 2011). La homogeneidad del medio, puede considerarse en principio una limitación, pero resaltamos la uniformidad cada vez más acusada de las noticias internacionales en los diarios españoles. Izquierdo, tras analizar las coincidencias temáticas en los contenidos de la sección internacional de El País, El Mundo, $A b c$ y La Razón, afirmó que: "la sección de Internacional presenta un elevado grado de coincidencia" (Izquierdo, 2012: 45). 
- Análisis de variables: la localización geográfica, la temática, las fuentes y la cobertura de las noticias son las variables estudiadas, mediante un análisis descriptivo. Para la localización, la referencia utilizada es la división geográfica del mundo por continentes. Para investigar las temáticas partimos de la metodología utilizada por Odriozola (2012: 162), que usa "los códigos propuestos por el International Press and Telecommunications Council para la transferencia internacional de noticias (Del Valle y García, 2002: 115)", a los que añade tres nuevas categorías: Tráfico, Sucesos y Otros. Y las fuentes se analizan a través de dos unidades de análisis: fuentes directas, aquellas elaboradas por el medio (firmadas por corresponsales, enviados especiales o por el propio medio); y fuentes indirectas (firmadas por agencias, o por otros medios ${ }^{2}$ ).

- Selección de la muestra: A través de la hemeroteca de elpais.es se han recogido las noticias aparecidas en su edición digital durante el primer trimestre del año $2012(\mathrm{n}=1.235)$. Todas las piezas informativas han sido analizadas para estudiar así de forma sistemática cada una de las variables, y poder controlar la cobertura informativa de cada uno de los acontecimientos.

- Interpretación de los resultados cuantitativos y conclusiones.

\section{Resultados}

\subsection{Localización de la información internacional}

$\mathrm{Si}$ atendemos a la localización de los acontecimientos internacionales, los datos muestran el siguiente porcentaje: América (42,4\%), Europa (26,4\%), Asia (23\%) y África $(8 \%)$. Sin apenas cobertura, Oceanía $(0,2 \%)$.

Tras analizar qué países tienen presencia mediática en cada uno de los continentes con cobertura informativa, los resultados obtenidos son los siguientes:

- Del continente americano han sido protagonistas en la prensa española los siguientes países, ordenados por orden decreciente: Estados Unidos (44\%), México $(13 \%)$, Argentina (8\%), Brasil (7,2\%), Venezuela (7\%), Colombia $(4,2 \%)$, Ecuador $(3,4 \%)$, Perú $(2,5 \%)$, Cuba $(2,4 \%)$, Guatemala y Chile (ambos con un $1,5 \%$ de representación), El Salvador (1,2\%), Honduras y Bolivia (ambos con un 1\%), Nicaragua $(0,8 \%)$, Costa Rica $(0,6 \%)$, Paraguay $(0,5 \%)$ y Haití $(0,2 \%)$. Por tanto, las regiones cubiertas son América del Norte y Latinoamérica. Mientras que de América del Norte interesa específicamente lo que acontece en EEUU, de Latinoamérica se informa prácticamente de todos los países.

EEUU tiene una enorme transcendencia internacional, sus decisiones e intervenciones influyen a escala global. Por ende, España mantiene vinculaciones culturales e históricas con los países latinoamericanos, y relaciones comerciales con algunos de ellos. Además, este diario muestra un interés especial por esta región geográfica al contar con ediciones que se imprimen y distribuyen en algunas de estas zonas. México,

2 En ocasiones el diario publica artículos obtenidos de otros periódicos internacionales, bien porque tenga acuerdos comerciales con ellos, o porque se les compra un artículo específico. En concreto, con el diario New York Times y Le Monde mantiene estos acuerdos desde 1998. 
país latinoamericano con mayor cobertura, es el principal destino de las exportaciones españolas, así como el principal proveedor de España. Argentina y Brasil, son también importantes proveedores y exportadores.

- Del continente asiático la información procede de los siguientes países: Siria (41,3\%), Irán (10,6\%), Israel y Afganistán (ambos con una representación de 8,8\%), China (8,5\%), Japón (5\%), Yemen e Irak (ambos con un 3,2 \%), Corea del Norte y Pakistán (los dos con un 2,1\% de representación), Pakistán (2,1\%), Kuwait y Taiwán (comparten un 1,4\%), Corea del Sur (1\%), Malasia $(0,7 \%)$, India $(0,7 \%)$, Líbano, Bahrein y Camboya (todos ellos con un $0,4 \%$ ). Ello indica que las zonas con más cobertura mediática son Asia del Este (se da cobertura a todos los países excepto a uno) y Medio Oriente (con representación casi la mitad de los países que componen esta zona geográfica). Más escasa es la presencia de Asia del Sur y Central.

Medio Oriente es, como sabemos, una zona conflictiva, donde tiene lugar la intervención de España para conseguir la estabilidad y el desarrollo de la región. Siria, país con mayor número de publicaciones, es una zona geoestratégica regional (su estabilidad implica la estabilidad futura de la región) $)^{3}$. Por otro lado, determinados países de Asia del Este, están emergiendo teniendo cada vez más transcendencia internacional por su importante desarrollo en diversos ámbitos; principalmente China, país con mayor número de informaciones de esta zona.

- Del continente europeo, los países que han acaparado la atención de la prensa española son de mayor a menor número de noticias: Francia $(28,4 \%)$, Italia $(11,9 \%)$, Bélgica (11,6\%), Reino Unido (10,4\%), Rusia (9,5\%), Alemania (7,2\%), Hungría, Grecia y Turquía (todos con una representación del 3\%), Rumanía (1,8\%), Holanda $(1,5 \%)$, Serbia y Montenegro (1,2\%), Islandia, Noruega y Portugal (con un $1 \%$ ), Suiza $(0,9 \%)$, Bielorrusia, Irlanda e Ucrania (todos con un 0,6\%), y Austria, Dinamarca, Eslovaquia, República Checa, Finlandia, junto a Polonia (todos con una representación del 0,3\%). La cobertura procede principalmente de Europa Occidental. La zona Oriental es cubierta en menor grado. Pero significativo es que el $68 \%$ de los países europeos con presencia mediática pertenecen a la Unión Europea.

Las decisiones adoptadas y los cambios que se producen en cada uno de los países que conforman la Unión Europa son de interés para España por sus posibles repercusiones. Los países con mayor cobertura de esta zona, Francia, Italia y Bélgica se caracterizan por ser los más próximos a España, y los que por su mayor peso económico son, junto a Alemania, parte del grupo motor de la Unión Europea. Rusia, país europeo de la zona Oriental con mayor cobertura, es una zona geográfica de interés, y además es un socio importante de la Unión Europea, puesto que junto a Estados Unidos y China, es uno de los que abastecen a este bloque.

- Del continente africano los países con presencia mediática son: Egipto (38 \%), Libia y Malí (los dos con 9\% de representación), Marruecos y Somalia (ambos con un $8 \%$ ), Nigeria y Senegal (comparten un 6\%), Túnez y Uganda (los dos con un 3\%), Sudán y Argelia (los dos un $2 \%$ ), y República del Congo, Etiopía, Liberia, Nueva

${ }^{3}$ Los acontecimientos posteriores han corroborado esta afirmación. 
Guinea, Sierra Leona, junto a Sudáfrica (todos tienen una representación del 1\%). Por tanto, tienen elevada cobertura los países que integran principalmente el Norte de África. Una zona caracterizada por tener conflictos reiterados, cuya inestabilidad afecta a los intereses de España, y al que se destina un elevado porcentaje de las exportaciones españolas. Egipto, zona con mayor cobertura, es un país geopolítico cuya estabilidad influye en el resto de países que conforman esta región africana.

Por tanto, podemos concretar qué zonas interesan más al medio analizado. Los circuitos comunicativos proceden de América del Norte, Latinoamérica, Medio Oriente, Asia del Este, zona Euro y Norte de África. Zonas de interés porque comprenden países que responden a uno o varios de los siguientes criterios:

1) Países con transcendencia internacional: aquellos que tienen un papel dominante en el contexto internacional, caracterizados por ser poderosos e influyentes, y los que empiezan a competir en el sistema internacional por su poder o influencia en sectores específicos a nivel mundial.

2) Países conflictivos: regiones que históricamente han vivido conflictos, o en las que se están produciendo tensiones o conflictos.

3) Países vinculados: caracterizados por tener lazos culturales, históricos, religiosos, económicos o políticos con España.

4) Países estratégicos: zonas de interés por su ubicación geográfica.

5) Países de interés geopolítico: zonas geoestratégicas que influyen en el resto de países que conforman su región geográfica.

Unos criterios que según como vaya configurándose la presencia, relaciones e intereses de España en el exterior, implicará que se modifique o no esta clasificación y los países que la integran, desapareciendo algunos o apareciendo nuevos actores.

Oceanía, al no contar con países que respondan a algunos de estos criterios, carece de cobertura informativa de forma sistemática.

\subsection{Temáticas de las noticias internacionales}

Las noticias internacionales informan sobre hechos vinculados a las siguientes temáticas: Política (37\%), Guerras-Conflictos (27,5\%), Asuntos sociales $(9,6 \%)$, Sucesos $(8,6 \%)$, Justicia (6,5\%), Economía-Negocios-Finanzas (6,2\%), Desastres y accidentes $(3,3 \%)$, y Religión-Creencias $(1,3 \%)$. Y, por tanto, las piezas informativas han versado, principalmente, sobre Política y Guerras-Conflictos. Datos que se corroboran al analizarlas por áreas geográficas.

Como se desprende del gráfico la sección internacional versa principalmente sobre política de ámbito obviamente internacional. Se trata de noticias que informan de hechos que pueden influir colateralmente en otros países (entre ellos España) o, por ende, en el propio país de origen. En concreto, los asuntos tratados en cada una de estas temáticas han sido los siguientes:

- En Política: elecciones (selección o designación de líderes políticos, campañas políticas, sondeos, mítines y referéndums); noticias de políticos (informaciones referentes a la esfera pública o privada de quienes se dedican a la política); medidas políticas (nuevas leyes, reformas constitucionales, aprobación de nuevos 
decretos ley, reformas de leyes, recortes, restricciones o censuras en el plano político, jurídico y social); relaciones internacionales o exteriores (actividades económicas, políticas, sociales y culturales que vinculan a un país con otro); y celebraciones políticas (festejos de acontecimientos políticos).

- En Guerras o Conflictos: conflictos armados y no militarizados; conflictos diplomáticos; tensiones políticas que puedan desencadenar en conflictos; autonomías e independentismos. Intervención del exterior en el conflicto.

Otros datos significativos obtenidos son los asuntos cubiertos en el resto de temáticas:

- Asuntos sociales: seguridad ciudadana (narcotráfico, salud pública y detenciones de personajes peligrosos para la humanidad); protestas sociales (huelgas, disturbios o manifestaciones); medios de comunicación (presentación de libros, programas de televisión, críticas realizadas en Webs, información sobre proyectos de comunicación, marketing, temas vinculados a medios a comunicación).

- Justicia: procesos judiciales (sentencias, resoluciones, acusaciones y detenciones por parte de la fiscalía, juicios, revisiones, muertes de personajes con procesos delictivos, delitos contra la humanidad, delitos militares o liberalizaciones).

- Economía: crisis económica (publicaciones que tratan sobre la marcha negativa de la economía de un país o área geográfica); medidas político-económicas (hechos que destacan por las medidas adoptadas en política fiscal, política de rentas, política de cambio y política monetaria por las autoridades económicas de un país); comercio internacional (intercambios de actividades comerciales entre países y empresas).

- Desastres y accidentes: tragedias marítimas, terrestres o aéreas; incendios producidos por agentes externos involuntarios; desastres ocasionados por fenómenos atmosféricos.

- Religión: celebraciones religiosas (festejos de acontecimientos religiosos); diferentes confesiones (acciones significativas de líderes religiosos); patrimonio eclesiástico (compensaciones aportadas a la iglesia católica por bienes incautados).

- Sucesos: Actos delictivos o violentos (informaciones sobre las formas emergentes de violencia, actos ilícitos premeditados y ejecutados).

Estos resultados muestran qué temáticas y asuntos interesan del exterior, y dónde se ha centrado principalmente la información. Aunque consideramos, que otros temas o asuntos podrían haber merecido también la atención de los medios.

\subsection{Cobertura de los acontecimientos}

Tras analizar el volumen de publicaciones de cada acontecimiento, los datos revelan que difiere la cobertura sobre temas que tratan de un mismo asunto. Lo que indica que el grado de intensidad en la información se rige por determinados criterios del medio. 
Los hechos con mayor presencia mediática han sido las elecciones de Estados Unidos (con 120 publicaciones), y el conflicto sirio (con 117 publicaciones). Con una presencia mediática muchísimo menos acusada, los acontecimientos cubiertos con más de diez publicaciones, son los expuestos en el siguiente gráfico: crisis económica en Europa, matanza de Toulousse, hundimiento del crucero Costa Concordia, visita del Papa a Latinoamérica, Enfermedad de Chávez, terremoto ocurrido en Fukushima, reforma sanitaria de Estados Unidos, y el proceso judicial de Rafael Correa contra el Universo.

Consideramos que el grado de cobertura depende de los siguientes criterios:

- La repercusión internacional o regional: hechos que afectan al orden internacional o estructura de una región específica.

- La cercanía: los que impliquen o afecten directamente a España.

- El grado de violencia o intensidad del acontecimiento.

- Lo inesperado e impactante: acontecimientos inesperados que provocan impacto social.

- Lo novedoso: acontecimientos que se producen por primera vez en una zona.

- Relación con el medio: hechos que tienen relación con los medios de comunicación o que puedan afectar en un futuro al propio medio.

\subsection{Las fuentes}

Lo que acontece en el mundo se nos cuenta principalmente a través de fuentes directas $(83,5 \%)$, noticias elaboradas por corresponsales principalmente, y ocasionalmente por enviados especiales. Son escasas las ocasiones que utilizan fuentes indirectas $(16,5 \%)$ y cuando recurren a ellas, suelen ser de agencia, a las que se añade información propia. Estos datos se confirman tras analizar las fuentes utilizadas en cada una de las áreas geográficas con cobertura informativa sistemática.

La credibilidad de las fuentes, y el intento del medio por buscar la mayor veracidad de la información, se aprecia en el tratamiento de las noticias internacionales. Los corresponsales viven en primera persona lo que sucede, y pueden discernir sobre el grado de credibilidad de otras informaciones, comprobar e interpretar los datos, y buscar la exclusividad (pueden ofrecer informaciones elaboradas exclusivamente para su medio de comunicación).

\section{Conclusiones}

- La cobertura informativa abarca Europa, Asia, África y América, con la menor presencia de Oceanía. Si bien, se centra en determinadas zonas: América del Norte, Latinoamérica, Unión Europea (Zona Euro), Medio Oriente, Asia del Este y Norte de África. Los países con más presencia mediática en cada una de estas zonas son Estados Unidos, México, Francia, Siria, China y Egipto.

- La información apunta en mayor medida hacia aquellos países que reúnen una o varias de las siguientes características: tener vinculaciones con España, tener influencia internacional, ser países emergentes, países estratégicos o de interés geopolítico.

- La información internacional trata, principalmente, sobre Política y Guerras- Conflictos. De cada una de estas temáticas interesan determinados asuntos. En política 
(elecciones, medidas políticas, noticias de políticos y relaciones internacionales o exteriores), y en guerras-conflictos (conflictos armados y no militarizados, conflictos diplomáticos, tensiones políticas, autonomías e independentismos). Noticias que informan de hechos que implican al mundo, a una región específica, o a España en particular.

- El resto de noticias pertenecen a las siguientes áreas temáticas: Asuntos sociales, Justicia, Economía-Negocios-Finanzas, Desastres y accidentes, Religión- Creencias. En cada una de ellas han predominado los siguientes temas. En EconomíaNegocios-Finanzas: la crisis económica y las medidas político económicas adoptadas; en Desastres y accidentes: tragedias (marítimas, terrestres o aéreas), incendios, fenómenos atmosféricos, etc.; en Religión- Creencias: noticias de líderes religiosos; en Asuntos Sociales: seguridad ciudadana, protestas sociales, medios de comunicación, etc.; en Justicia: procesos judiciales; y en Sucesos: actos delictivos y/o violentos.

- Los acontecimientos que tratan de un mismo asunto no son tratados con la misma cobertura informativa. Determinados criterios puede que condicionen su grado de intensidad en el medio: repercusión internacional o regional, cercanía, grado de intensidad, acontecimiento inesperado e impactante, hechos novedosos, relación con el medio.

- Las informaciones se elaboran sobre todo a través de fuentes directas. El medio invierte para contar con recursos propios que le permitan informar desde el lugar de los hechos. Escasas son las ocasiones que han utilizado informaciones de segunda mano, elaboradas por otros medios. Y cuando las han utilizado, se caracterizan por contrastar la información con otras agencias y añadir información propia.

- Las aportaciones de esta investigación permiten conocer la agenda y la geografía de la información internacional. Parece necesario seguir profundizando en estos aspectos diversificando las fuentes de la investigación y proponiendo estudios comparados. El estudio de los acontecimientos internacionales y de su contexto social, político, económico o cultural, y su reflejo en los medios de comunicación de forma pormenorizada nos proporcionarán las claves para entender el mundo que nos rodea. La radiografía presentada en el trabajo actual nos permitirá focalizar con precisión nuevas líneas de investigación.

\section{Referencias bibliográficas}

BADIOU, Alain (1988): L'être et L'événement. Paris, Seuil.

CAMPOS, María Victoria y MARTÍNEZ, Fátima (2012): "La información internacional en la prensa diaria española: los criterios de los redactores jefes", en DE CUETO, Carlos y CALATRAVA, Adolfo: Defensa y Globalización. Granada, Editorial Universidad de Granada, pp. 535-548.

COMSCORE (2012): "El uso de Internet en Europa", en comScore: http://www.comscore.com/es1/layout/set/popup/Press_Events/Press_Releases/2012/1/Nearly_50_Percent_of_Internet_Users_in_Europe_Visit_Newspaper_Sites, 19-1-2012. [fecha de consulta: 3 de marzo de 2011]. 
DE LA CALLE, Jaime (2010): "Para una teoría social del acontecimiento". Athenea Digital, n 18 , pp. 65- 81 .

DEL VALLE, Félix, y GARCÍA, Antonio (2002): "Construcción de un tesauro para el Centro de Documentación de Telecinco". Scire, no 8 (1), pp.103-118.

DÍAZ, Rafael (2008): "La representación del mundo en los informativos de televisión". Estudios del Mensaje Periodístico, vol. 14, pp. 363- 384. Madrid, servicio de Publicaciones de la Universidad Complutense.

FONTANA, Josep (1992): La Historia después del fin de la Historia. Barcelona, Crítica.

IZQUIERDO, Luis (2012): "La uniformidad temática en las secciones de internacional a los medios madrileños de información general frente a las secciones locales". index.comunicación, $\mathrm{n}^{\mathrm{0}}$ 2, pp.37-48.

McCOMBS, Maxwell (2006): Estableciendo la agenda: el impacto de los medios en la opinión pública y el conocimiento. Barcelona, Paidós.

MEAD, George Herbert (1932): La filosofia del presente. Traducción de la edición original, póstuma (Chicago, 1932), http://www.unav.es/gep/FilosofiaPresente.pdf.

MOLES, Abraham (1972): "Notes pour une typologie des événements". Comunications, v. 18, nº 18, pp. 90-96.

MORENO, Pastora (2000): "Los géneros periodísticos informativos en la actualidad internacional". Ámbitos, vol. 5: http:/www.ull.es/publicaciones/latina/ambitos/5/35moreno.htm

MORÍN, Edgar (1972): "L'événement-sphinx”. Communications, v. 18, nº 18, pp.173192.

NAVARRO, Lizy (2002): Los periódicos on line. México, Universitaria Potosina.

ODRIOZOLA, Javier (2012): "Cibermedios y agenda setting: la configuración de la agenda mediática internacional”. Estudios sobre el Mensaje Periodístico, vol. 18, $\mathrm{n}^{\circ}$ 1, pp.157-171. Madrid, Servicio de Publicaciones de la Universidad Complutense.

TUDESQ, André-Jean (1973): La presse et l'évènement. Paris, Mouton.

VERÓN, Eliseo (1983): Construir el acontecimiento. Barcelona, Gedisa. 